\title{
BCS-Spin-Model, its Thermodynamic Representations and Automorphisms
}

\author{
F. JELINEK \\ Institute for Theoretical Physics, University of Vienna \\ Received April 1, 1968
}

\begin{abstract}
In this paper two equivalent explicit realizations of the thermodynamic representations of the BCS-spin-model are constructed and since they are of well known form their type is easily determined. Time evolution and vacuum properties of these representations are studied and it is shown that in general the time development of the system is representation-dependent.
\end{abstract}

Our starting point is the Hamiltonian

$$
H_{N}=\varepsilon \sum_{p=1}^{N}\left(1-\sigma_{p}^{z}\right)-\frac{2 T_{0}}{N} \sum_{p p^{\prime}=1}^{N} \sigma_{p}^{-} \sigma_{p^{\prime}}^{+}
$$

where the first term describes the energy of Cooper-pairs and the second their interaction. $\left(\sigma_{p}=\left(\sigma_{p}^{x}, \sigma_{p}^{y}, \sigma_{p}^{z}\right)\right.$ are the usual Pauli matrices $\left.\sigma_{p}^{ \pm}=\left(\sigma_{p}^{x} \pm i \sigma_{p}^{y}\right) / 2\right)$. For $N \rightarrow \infty$ the thermal expectation values of products of $\boldsymbol{\sigma}_{p}$ 's simplify and just these limits are used for physical calculations. The price one has to pay for this simple structure of the thermodynamic functional are the mathematical difficulties connected with infinite particle numbers.

Let $\Sigma_{1}$ be the smallest *-algebra generated by the $\boldsymbol{\sigma}_{p}$ 's. We suppose the elements of $\Sigma_{1}$ to be realized in a natural way as bounded linear operators in the Hilbert-space $\mathfrak{G}=\bigotimes_{p=1}^{\infty} \mathfrak{G}_{p}$ (all $\mathfrak{H}_{p}$ two-dimensional) [1]. In this representation one takes the uniform closure (in the sense of the usual operator norm $\left.\|A\|=\sup _{\|x\|=1}\|A x\| x \in \mathcal{S}\right)$ of $\Sigma_{1}$, we call the resulting $C^{*}$-algebra $\Sigma$.

Now we state a result: Let $A$ be an element of $\Sigma_{1}$ and

$$
\langle A\rangle_{\beta}=\lim _{N \rightarrow \infty}\langle A\rangle_{N, \beta}=\lim _{N \rightarrow \infty} \frac{\operatorname{tr}\left(e-\beta H_{N} A\right)}{\operatorname{tr}\left(e^{\left.-\beta H_{N}\right)}\right.}
$$

where the trace for fixed $N$ goes over the $2^{N}$-dimensional space on which the (only) irreducible representation of the system $\left\{\boldsymbol{\sigma}_{1}, \ldots \boldsymbol{\sigma}_{N}\right\}$ acts. For $\beta \leqq \beta_{0}=\varepsilon^{-1}$ arcth $\varepsilon / T_{0}$ one gets

$$
\left\langle\sigma_{p_{1}}^{\left(i_{1}\right)} \ldots \sigma_{p_{m}}^{\left(i_{m}\right)}\right\rangle_{\beta}=(\operatorname{th} \beta \varepsilon)^{m} n^{\left(i_{1}\right)} \ldots n^{\left(i_{m}\right)} \text { with } \boldsymbol{n}=(0,0,1)
$$

12 Commun. math. Phys., Vol. 9 
and for $\beta>\beta_{0}$

$$
\begin{gathered}
\left\langle\sigma_{p_{1}}^{\left(i_{1}\right)} \ldots \sigma_{p_{m}}^{\left(i_{m}\right)}\right\rangle_{\beta}=\int_{0}^{2 \pi} \frac{d \phi}{2 \pi}\left\langle\sigma_{p_{1}}^{\left(i_{1}\right)} \ldots \sigma_{p_{m}}^{\left(i_{m}\right)}\right\rangle_{\beta, \phi} \text { where } \\
\left\langle\sigma_{p_{1}}^{\left(i_{1}\right)} \ldots \sigma_{p_{m}}^{\left(i_{m}\right)}\right\rangle_{\beta, \phi}=y^{m} n^{\left(i_{1}\right)} \ldots n^{\left(i_{m}\right)} \quad \text { with } \\
\boldsymbol{n}=\left(\cos \phi \sqrt{1-\left(\frac{\varepsilon}{T_{0} y}\right)^{2}}, \quad \sin \phi \sqrt{1-\left(\frac{\varepsilon}{T_{0} y}\right)^{2}}, \frac{\varepsilon}{T_{0} y}\right)
\end{gathered}
$$

and $y$ fulfils the equation arcth $y=\beta T_{0} y$ (see appendix). The functional \langle\rangle$_{\beta}$ is linear and positive on $\Sigma_{1}$ and can uniquely be extended to $\Sigma$. We now construct the corresponding representations $\pi_{\beta}(\Sigma)$.

First we consider \langle\rangle$_{\beta, \phi}\left(\beta>\beta_{0}\right)$. Its product property suggests a realization of the corresponding representation in an infinite tensor product of Hilbert-spaces. This is actually possible in the following way: Let be $\mathfrak{Y}=\bigotimes_{p=1}^{\infty} \mathfrak{o} \overline{\mathfrak{G}}_{p}$ an incomplete direct product space, each $\overline{\mathfrak{G}}_{p}$ being four-dimensional. Such an $\overline{\mathfrak{Y}}_{p}$ can obviously be written as $\overline{\mathfrak{Y}}_{p} \cong \mathfrak{H}_{p}^{(1)} \otimes \mathfrak{G}_{p}^{(2}$ with $\mathfrak{H}_{p}^{(i)}$ two-dimensional. We take the strong equivalence class $\mathfrak{P}$ to be determined by the vector

$$
\left.\left.\left.\left.\xi_{\beta \phi}=\bigotimes_{p=1}^{\infty}\left(\sqrt{\frac{1+y}{2}} \mid \boldsymbol{n}\right) \otimes \mid \boldsymbol{n}\right)+\sqrt{\frac{1-y}{2}} \mid-\boldsymbol{n}\right) \otimes \mid-\boldsymbol{n}\right)\right)_{p=1}^{\infty}(\ldots)_{p}
$$

$(\boldsymbol{n})$ is the vector which is determined uniquely up to a phase-factor by the relation $(\boldsymbol{\sigma n}) \mid \boldsymbol{n})=\mid \boldsymbol{n})$.

Now take $\pi_{\beta, \phi}\left(\sigma_{p}\right)=\left(\bigotimes_{q \neq p}(1 \otimes 1)_{q}\right) \otimes(\sigma \otimes 1)_{p}$. One easily convinces oneself that for all $A \in \Sigma_{1}$

$$
\left\langle\xi_{\beta, \phi} \mid \pi_{\beta, \phi}(A) \xi_{\beta, \phi}\right\rangle=\langle A\rangle_{\beta, \phi},
$$

if $\boldsymbol{n}$ and $y$ are chosen to be the same as in (4) and that $\xi_{\beta, \phi}$ is actually a cyclic vector of $\pi_{\beta, \phi}\left(\pi_{\beta, \phi}\left(\Sigma_{1}\right) \xi_{\beta, \phi}\right.$ dense in $\left.\mathcal{H}\right)$. According to [2] $\left(\pi_{\beta, \phi}(\Sigma)\right)^{\prime \prime}$ is a factor of type III for $0<y<1$ and a factor of type $\mathrm{II}_{1}$ for $y=0$. For $y=1(\beta=\infty)$ the representation is irreducible and acts in $\bigotimes_{p=1}^{\infty} \mathfrak{G} \mathfrak{H}_{p}^{(i)}$ with $\mathfrak{c}$ determined by $\left.\xi=\bigotimes_{p=1}^{\infty} \mid \boldsymbol{n}\right)_{p}$.

For the case $0 \leqq y<1$ another equivalent realization exists: Let $S_{\boldsymbol{n}}$ be the abelian $C^{*}$-subalgebra in $\Sigma$, generated by the $\left\{\left(\boldsymbol{\sigma}_{p} \boldsymbol{n}\right)\right\}, \Gamma$ the set of all sequences $\varepsilon=\left\{\varepsilon_{p}\right\}$ with $\varepsilon_{p}=0$ or 1 equipped with the product topology of the discrete topology of components. We define an addition by componentwise addition $(\bmod 2)$. Let $G$ be the countable discrete subgroup of $\Gamma$ consisting of sequences with only a finite number of $\varepsilon_{p}=1$ and $\nu$ its Haar-measure. Let further be $\mu_{(1+y) / 2}$ the regular product measure on $\Gamma$ with $\mu_{(1+y) / 2}(\Gamma)=1$ and $\mu_{(1+y) / 2}\left(\left\{\varepsilon: \varepsilon_{p}=0\right)\right\}=(1+y) / 2$ for all $p$. Then the assertion is that an equivalent realization of $\pi_{\beta, \phi}(\Sigma)$ acts in a Hilbertspace $L^{2}(G, v) \otimes L^{2}\left(\Gamma, \mu_{(1+y) / 2}\right)$ consisting of square-integrable functions 
$f(\gamma, \varepsilon) \gamma \in G, \varepsilon \in \Gamma$. Now let $\boldsymbol{n}_{\mathbf{1}}, \boldsymbol{n}_{\mathbf{2}}$ be two normed vectors orthogonal to each other and to $\boldsymbol{n}$. The generating elements of $\Sigma$ now act as:

$$
\begin{aligned}
\pi\left(\left(\boldsymbol{\sigma}_{p} \boldsymbol{n}\right)\right) f(\gamma, \varepsilon) & =(-1)^{\varepsilon_{p}} f(\gamma, \varepsilon) \\
\pi\left(\left(\boldsymbol{\sigma}_{p} \boldsymbol{n}_{1}\right)\right) f(\gamma, \varepsilon) & =\left(\frac{1-y}{1+y}\right)^{\frac{1}{2}\left(1-2 \varepsilon_{p}\right)} f\left(\gamma+\delta_{p}, \varepsilon+\delta_{p}\right) \\
\pi\left(\left(\boldsymbol{\sigma}_{p} \boldsymbol{n}_{2}\right)\right) f(\gamma, \varepsilon) & =-i(-1)^{\varepsilon_{p}}\left(\frac{1-y}{1+y}\right)^{\frac{1}{2}\left(1-2 \varepsilon_{p}\right)} f\left(\gamma+\delta_{p}, \varepsilon+\delta_{p}\right) \\
\delta_{p} & =(0, \ldots 0,1,0,0 \ldots) .
\end{aligned}
$$

Type and factor property of this representation now follow from the well known theorems of v. Neumann [3].

Collecting our findings we can state the assertion: For the thermodynamic representation $\left(\pi_{\beta}(\Sigma)\right)^{\prime \prime}$ is for

$\beta=\infty \quad$ a direct integral of irreducible representations

$\beta_{0}<\beta<\infty$ a direct integral of type III-factors

$\beta_{0} \geqq \beta>0 \quad$ a factor of type III

$\beta=0 \quad$ a factor of type $\mathrm{II}_{1}$.

Now we turn to the time-development described by our Hamiltonian. One sees easily that quantities $e^{i H_{N} t} A e^{-i H_{N} t}=A_{N}(t) A \in \Sigma$ cannot converge uniformly for $N \rightarrow \infty$, for that would imply uniform convergence in all representations to the same time-development. As a matter of fact the above $A_{N}(t)$ converges 1 . only in certain representations of $\Sigma, 2$. not uniformly but only strongly and 3 . in different representations to different limits. More concrete and fairly elementary one can show the following theorem [4]. Let $\pi(\Sigma)$ be a representation where $\frac{1}{N} \sum_{1}^{N} \boldsymbol{\sigma}_{p} \rightarrow \eta \boldsymbol{s}$, $0 \leqq \eta \leqq 1, \eta, s$ diagonal. Then

$$
\begin{aligned}
& e^{i H_{N} t} \boldsymbol{\sigma}_{p} e^{-i H_{N} t} \stackrel{\text { strongly }}{\longrightarrow}\left[\cos 2 t\left(\eta T_{0} s^{z}-\varepsilon\right)+\left(1-\cos 2 t\left(\eta T_{0} s^{z}-\varepsilon\right)\right)\right. \\
& \left.\boldsymbol{\mu}_{\circ} \boldsymbol{\mu}+\sin 2 t\left(\eta T_{0} s^{z}-\varepsilon\right) \boldsymbol{\mu} \times\right]\left[\cos 2 \eta T_{0} t+\left(1-\cos 2 \eta T_{0} t\right) \boldsymbol{s}_{\circ} \boldsymbol{s}\right. \\
& \left.\quad-\sin 2 \eta T_{0} t \boldsymbol{s} \times\right] \boldsymbol{\sigma}_{p}, \quad \boldsymbol{\mu}=(0,0,1) .
\end{aligned}
$$

In these representations the time development is a combined rotation around the axes $\boldsymbol{\mu}$ and $\boldsymbol{s}$. Obviously the above formula defines an automorphism of the respective $C^{*}$-algebras $\pi(\Sigma)$ if one extends from $\pi\left(\Sigma_{1}\right)$ in the usual way. As $\Sigma$ is simple all its nontrivial representations are faithful and one can attach to each automorphism $\bar{\tau}_{\pi}$ in a representation $\pi(\Sigma)$ an automorphism $\tau_{\pi}$ of $\Sigma$ by putting $\tau_{\pi} x=\pi_{\circ} \bar{\tau}_{\pi \circ} \pi^{-1}(x)$ for all $x \in \Sigma$. In our case $\tau_{\pi}$ is evidently representation-dependent, a fact which stems from the great interaction range in our Hamiltonian. It is also the reason why in certain representations particle-number and timeautomorphism do not commute. (The particle-number automorphism is $12^{*}$ 
the transformation $\tau_{\phi} \boldsymbol{\sigma}_{p}=\left(\cos \phi+(1-\cos \phi) \boldsymbol{\mu}_{\circ} \boldsymbol{\mu}+\sin \phi \boldsymbol{\mu} \times\right) \boldsymbol{\sigma}_{p}$ extended to $\Sigma$.) This strange situation in view of commutativity of the local generators $H_{N}$ and $K_{N}=\sum_{1}^{N} \sigma_{p}^{z}$ is caused by the fact that $e^{i H_{N} t} A e^{-i H_{N} t}=e^{\mathrm{itad} H_{N}} A$, ad $H_{N} A=\left[H_{N}, A\right]$, does not converge uniformly as $N \rightarrow \infty$.

One can show elementary that $e^{\text {it ad } H_{N}} \rightarrow \alpha(t)$ and $e^{i \Phi \text { ad } K_{N}} \rightarrow \beta(\phi)$ as $N \rightarrow \infty$ and $\left[H_{N}, K_{N}\right]=0$ implies $[\alpha, \beta]=0(\rightarrow$ means here strong convergence of operators on the Banach-space $\Sigma$ ). As the strong convergence of terms $e^{i t a d H_{N}}$ is technically difficult to handle for spin systems, it is advantageous to reduce the problem to convergence of ad $H_{N}$ on a dense set in $\Sigma$ :

Theorem: Let $\left\{H_{N}\right\}$ be a sequence of Hamiltonians (for fixed $N$ they depend on $\left.\boldsymbol{\sigma}_{1} \ldots \boldsymbol{\sigma}_{N}\right)$. Then $\operatorname{ad} H_{N} \rightarrow A$ on a dense set in $\Sigma$ implies $e^{\text {itad } H_{N}} \rightarrow e^{\text {it } A}$ on $\Sigma$. We sketch the proof: As all the $H_{N}$ are hermitian the resolvent set of $\operatorname{ad} H_{N}$ is real, therefore $\frac{1}{\lambda-i \operatorname{ad} H_{N}}=J_{N}(\lambda)$ is a uniform proper resolvent family which converges strongly on $\Sigma$ because of our assumption $J_{N}(\lambda) \rightarrow J(\lambda)$. This implies ([5] Theorem 5.1) $e^{\text {it ad } H_{N}}$ $\rightarrow e^{\text {it } A}$ on $\Sigma$.

Strong convergence of the derivations ad $H_{N}$ on a dense subset of $\Sigma$ is therefore a useful sufficient condition for existence of a unique automorphism of the $C^{*}$-algebra $\Sigma$. It allows to show this uniqueness easily for Heisenberg and Ising models with appropriate interaction range. It suffices to show convergence of ad $H_{N} \boldsymbol{\sigma}_{N}$ which implicates convergence on the dense set $\Sigma_{1}$.

Finally we regard time-development in thermodynamic representations. For $\beta_{0}>\beta>0$ we have $\pi_{\beta}\left(\frac{1}{N} \sum_{1}^{N} \boldsymbol{\sigma}_{p}\right) \rightarrow y \boldsymbol{n}$ and we can therefore define an automorphism. Every automorphism of a type III-algebra is spatially representable [6]. If one postulates uniqueness of the vacuum, the corresponding unitary operator cannot be an element of $\left(\pi_{\beta}(\Sigma)\right)^{\prime \prime}$. In the incomplete direct product space-representation it has the form:

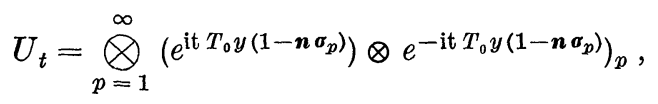

to postulate uniqueness of vacuum seems to make sense as the thermodynamic functional has a strong cluster decomposition property:

$$
\left\langle\sigma_{p_{1}}^{\left(i_{1}\right)}\left(t_{1}\right) \ldots \sigma_{p_{n}}^{\left(i_{n}\right)}\left(t_{n}\right)\right\rangle_{\beta}=\left\langle\sigma_{p_{1}}^{\left(i_{1}\right)}(0)\right\rangle_{\beta} \ldots\left\langle\sigma_{p_{n}}^{\left(i_{n}\right)}(0)\right\rangle_{\beta}
$$

(that the functional decomposes for all values of $t$ is a speciality of the model). 
Below the critical temperature, i.e. $\beta_{0}<\beta<\infty$, a unitary timedevelopment operator with unique vacuum cannot exist as there is not even a weak cluster-decomposition property. A sequence of orthogonal vacua is for example $\int^{\oplus} e^{\text {in } \phi} \xi_{\beta, \phi} d \phi / 2 \pi, n$ integer. The (factor) decomposition of the thermodynamic representations corresponds exactly to the decomposition of an invariant functional into extremal invariant functionals [7]. In this relation the BCS-spin model seems to be representative for systems which below some temperature have a transition to a phase of higher order.

Acknowledgements. I wish to thank Prof. W. Thirking for posing the problem and Dr. A. WeHrL for helpful discussions.

\section{Appendix}

Calculation of thermodynamic expectation values $[8,9]$. We calculate a generating functional which by differentiation in parameters $a$, $b, c$ yields all expectation values of elements $\varepsilon \Sigma_{1}$

$$
\begin{aligned}
& \langle A(a, b, c)\rangle_{\beta}=\lim _{N \rightarrow \infty} \frac{\operatorname{tr} e-\beta H_{N} A_{N}(a, b, c)}{\operatorname{tr} e^{-\beta H_{N}}}=\lim _{N \rightarrow \infty}\left\langle A_{N}\right\rangle_{N, \beta} \\
& A_{N}(a, b, c)=e^{i \frac{a}{N} \sum_{1}^{N} \sigma_{p}^{x}} e^{i \frac{b}{N} \sum_{1}^{N} \sigma_{p}^{y}} e^{i \frac{c}{N} \sum_{1}^{N} \sigma_{p}^{z}} \text {, call } \sum \boldsymbol{\sigma}_{p}=2 \boldsymbol{L} \\
& \operatorname{tr} e^{-\beta H_{N}} A_{N}=\sum_{L=0}^{N / 2} \sum_{L^{z}=-L}^{L} \frac{N !(2 L+1)}{(N / 2-L) !(N / 2+L+1) !} \\
& \cdot e^{\beta\left[\varepsilon\left(2 L^{z}-N\right)+\frac{2 T_{0}}{N}\left(L(L+1)-L^{z}\left(L^{z}+1\right)\right)\right]} \cdot G_{N}\left(\frac{2 L}{N}, \frac{2 L^{z}}{N} ; a, b, c\right) \\
& \operatorname{tr} e^{-\beta H_{N}}=\sum_{L=0}^{N / 2} \sum_{L^{\varepsilon}=-L}^{L} \frac{N !(2 L+1)}{(N / 2-L) !(N / 2+L+1) !} \\
& \cdot e^{\beta\left[\varepsilon\left(2 L^{z}-N\right)+\frac{2 T_{0}}{N}\left(L(L+1)-L^{z}\left(L^{z}+1\right)\right)\right]} \\
& G_{N}\left(\frac{2 L}{N}, \frac{2 L^{z}}{N} ; a, b, c\right)=\left\langle L L^{z}\left|A_{N}(a, b, c)\right| L L^{z}\right\rangle \\
& =\left\langle L L^{z}\left|e^{2 i \frac{a}{N} L^{x}} e^{2 i \frac{b}{N} L^{y}} e^{2 i \frac{c}{N} L^{z}}\right| L L^{z}\right\rangle \\
& =\sum_{\chi}\left(\begin{array}{c}
L+L^{z} \\
\chi
\end{array}\right)\left(\begin{array}{c}
L-L^{z} \\
\chi
\end{array}\right) u_{11}^{L+L^{z}-\chi} u_{12}^{\chi} u_{21}^{\chi} u_{22}^{L-L^{z}-\chi} \\
& u_{11}=\left[\cos \frac{a}{N} \cos \frac{b}{N}-i \sin \frac{a}{N} \cos \frac{b}{N}\right] e^{i \frac{c}{N}} \\
& u_{12}=\left[\cos \frac{a}{N} \sin \frac{b}{N}+i \sin \frac{a}{N} \cos \frac{b}{N}\right] e^{-i \frac{c}{N}} \\
& u_{21}=\left[i \sin \frac{a}{N} \cos \frac{b}{N}-\cos \frac{a}{N} \sin \frac{b}{N}\right] e^{i \frac{c}{N}} \\
& u_{22}=\left[i \sin \frac{a}{N} \sin \frac{b}{N}+\cos \frac{a}{N} \cos \frac{b}{N}\right] e^{-i \frac{c}{N}} .
\end{aligned}
$$


In $G_{N}$ let $N$ go to infinity, $\frac{2 L}{N} \rightarrow y_{0} \frac{2 L^{z}}{N} \rightarrow w_{0}$. Then $G_{N}\left(\frac{2 L}{N}, \frac{2 L^{z}}{N} ; a, b, c\right) \rightarrow e^{i w_{0} c} J_{0}\left(\sqrt{\left(y_{0}^{2}-w_{0}^{2}\right)\left(a^{2}+b^{2}\right)}\right)=G_{\infty}\left(y_{0}, w_{0} ; a, b, c\right)$.

Setting $\frac{2 L}{N}=y, \frac{2 L^{z}}{N}=w$ one can write (13) as a Stieltjes-integral: $\left\langle A_{N}\right\rangle_{N, \beta}=\int G_{N}(y, w ; a, b, c) d \mu_{N}(y, w)$ over the triangle $0 \leqq y \leqq 1$, $|w| \leqq y$. As $N \rightarrow \infty$ the measures $\mu_{N}$ tend to a point-measure concentrated in $\left(y_{0}, w_{0}\right)$ with arcth $y_{0}=\beta T_{0} y_{0}$ and $w_{0}=\varepsilon / T_{0}$. Because of the uniform convergence of the $G_{N}$ one concludes $\left\langle A_{N}\right\rangle_{N, \beta} \stackrel{N \rightarrow \infty}{\longrightarrow}$ $G_{\infty}\left(y_{0}, w_{0} ; a, b, c\right)$. This is only right for $\beta \geqq \varepsilon^{-1} \operatorname{arcth} \varepsilon / T_{0}=\beta_{0}$.

For $\beta<\beta_{0}$ the measures get concentrated on $\left(y_{0}, w_{0}\right)$ with $y_{0}=w_{0}$ $=$ th $\beta \varepsilon$ and $\left\langle A_{N}\right\rangle_{N, \beta} \rightarrow e^{\text {icth } \beta \varepsilon}$. Therefore in this case

$$
\left\langle\sigma_{p_{1}}^{\left(i_{1}\right)} \ldots \sigma_{p_{m}}^{\left(i_{m}\right)}\right\rangle=(\operatorname{th} \beta \varepsilon)^{m} n^{\left(i_{1}\right)} \ldots n^{\left(i_{m}\right)} \quad \boldsymbol{n}=(0,0,1) .
$$

For $\beta>\beta_{0}$ one can decompose the functional:

$$
\begin{aligned}
\langle A\rangle_{\beta} & =\int_{0}^{2 \pi} \frac{d \phi}{2 \pi}\langle A\rangle_{\beta, \phi} \\
\langle A\rangle_{\beta, \phi} & =\lim _{N \rightarrow \infty}\left\langle A_{N}(a, b, c)\right\rangle_{N, \beta, \phi}=\lim _{N \rightarrow \infty} \frac{\operatorname{tr} e^{-\beta H_{N, \phi}} A_{N}}{\operatorname{tr} e^{-\beta H_{N}, \phi}}
\end{aligned}
$$

$H_{N, \phi}=-\beta^{-1} \omega \sum_{p=1}^{N}\left(\boldsymbol{\sigma}_{p} \boldsymbol{n}\right), \quad \boldsymbol{n}=(\cos \phi \sin \theta, \sin \phi \sin \theta, \cos \theta)$

$\langle A(a, b, c)\rangle_{N, \beta, \phi}$

$$
=\left\{1+i \frac{\text { th } \omega}{N}(a \cos \phi \sin \theta+b \sin \phi \sin \theta+c \cos \theta)+0\left(N^{-2}\right)\right\}^{N}
$$

$$
\underset{N \rightarrow \infty}{\longrightarrow} e^{i \operatorname{th} \omega(\mathrm{a} \cos \phi \sin \theta+\mathrm{b} \sin \phi \sin \theta+\mathrm{c} \cos \theta)}=\langle A\rangle_{\beta, \phi}
$$

$\int_{0}^{2 \pi} \frac{d \phi}{2 \pi}\langle A\rangle_{\beta, \phi}=J_{0}\left(\operatorname{th} \omega \sin \theta \sqrt{\left.a^{2}+b^{2}\right)} e^{\mathrm{ic} \operatorname{th} \omega \cos \theta}=\langle A(a, b, c)\rangle_{\beta}\right.$

by comparison one determines $\omega, \theta$ :

th $\omega \sin \theta=\sqrt{y_{0}^{2}-w_{0}^{2}}$ th $\omega \cos \theta=w_{0} ;$ th $\omega=y_{0} \quad \cos \theta=\frac{w_{0}}{y_{0}}$.

Therefore

$$
\left\langle\sigma_{p_{1}}^{i_{1}} \ldots \sigma_{p_{m}}^{i_{m}}\right\rangle_{\beta}=\int_{0}^{2 \pi} \frac{d \phi}{2 \pi} y_{0}^{m} n^{i_{1}} \ldots n^{i_{m}}
$$




\section{References}

1. Neumann, J. v.: Collected Works III. . On infinite direct products. New York: Pergamon 1961.

2. Bures, D. J. C.: Compositio Math. 32, 169 (1964).

3. NeumanN, J. v.: Collected Works III. On rings of operators III. New York: Pergamon 1961.

4. JeLINEK, F.: Thesis, Vienna (1968).

5. Trotter, H. F.: Pacific J. Math. 8, 887 (1958).

6. Dixmier, J.: Les algèbres d'operateurs dans l'espace hilbertien. Paris: GauthierVillars 1957.

7. Ruelle, D.: Commun. Math. Phys. 3, 133 (1966).

8. Thirring, W., and A. Wehri: Commun. Math. Phys. 4, 1 (1967).

9. - Commun. Math. Phys. 7, 181 (1968).

F. JELINEK

Institut f. theoret. Physik der Universität A 1090 Wien, Boltzmanngasse 